\title{
Resistance and AC Susceptibility of YBCO 1:2:3 Films on Silver Substrates
}

\author{
R. Zalecki, W.M. WoCh*, M. Chrobak and A. Ko£odziejczyk \\ Solid State Physics Department, AGH University of Science and Technology \\ Mickiewicza 30, 30-059, Kraków, Poland
}

The $\mathrm{YBa}_{2} \mathrm{Cu}_{3} \mathrm{O}_{\delta}$ films were obtained directly on the polished silver substrates by the sedimentation process. Thickness of these films is of the order of several tens micrometers. The temperature dependences of the resistance and the AC susceptibility of the films were measured and analyzed. The critical temperatures of these specimens vary from $89 \mathrm{~K}$ to $91 \mathrm{~K}$ and they do not depend on the annealing temperatures. The critical currents were calculated from AC susceptibility measurements using the Bean's model.

PACS: 74.72.-h, 74.25.F, 74.25.Ha, 74.25.Sv

\section{Introduction}

Silver foils are typically used as a coat of superconducting material in superconducting tapes and wires, particularly of the thallium or bismuth based high temperature superconductors [1-3]. They may be also used as the substrates for superconducting films. The direct deposition of YBCO films on silver substrates without any buffer layers is one of the most promising and low-cost method of production of the superconducting tapes [4]. Silver turned out to be the only metal on which YBCO can be deposited without any buffer layers because it does not react with YBCO and fills the pores and empty spaces between the grains [5]. However, the silver substrate may influence the deposited superconducting material due to the sublimation processes of silver at the high sintering temperatures as well as due to crystal mosaics which are formed on a silver surface at high temperatures. This problem is particularly important in the case of YBCO high temperature superconductors because they require high sintering temperatures up to $950^{\circ} \mathrm{C}$ to obtain good superconducting quality properties. Unfortunately, our attempts to sinter the YBCO films on the Ag substrate at such high temperature failed, due to a damage of the layer. On the other hand, the thallium and particularly BiSCO superconductors can be typically sintered at lower temperatures and silver is successfully applied for manufacturing the superconducting tapes [6]. Silver also enables using high pressures for pressing the superconductors, which is very important for achieving the good quality polycrystalline superconductor.

In this paper we present results of resistance and $\mathrm{AC}$ susceptibility measurements of YBCO thick films deposited on silver substrate and sintered at temperatures of $925^{\circ} \mathrm{C}$ and $915^{\circ} \mathrm{C}$. The thickness of the films is about $90 \mu \mathrm{m}$ and $80 \mu \mathrm{m}$ respectively which is typical for the superconducting tapes rather than for the superconducting films. The aim of this work is to examine the properties

\footnotetext{
* corresponding author; e-mail: wmwoch@agh.edu.pl
}

of YBCO superconductors sintered at lowered temperatures and deposited on silver substrate.

\section{Sample preparation}

YBCO polycrystalline samples were prepared with the standard powder solid-state reaction technique. It consists of mixing of the copper and yttrium oxides and the barium carbonate in appropriate amounts. The mixed powders were divided into two parts which were sintered at $925^{\circ} \mathrm{C}$ or at $915{ }^{\circ} \mathrm{C}$ respectively. They were next treated separately. After sintering they were cooled down in a flowing oxygen to the temperature of $400^{\circ} \mathrm{C}$ within the period of $4 \mathrm{~h}$. After the one hour oxidation the sample was cooled down to the room temperature in $2 \mathrm{~h}$. Both fractions were two times re-grinded and re-sintered. The heat treatment was repeated separately for each of these parts to prepare the pure phase powder. After the final regrinding, the powder was pressed into the pallets with the pressure of $1 \mathrm{GPa}$. The rest of the YBCO powder was used to form the YBCO thick films on the silver substrate.

YBCO powder was mixed in the isopropanol to make the powder suspension in alcohol. Then it was left for 10 minutes and after the largest grains had fallen down only the suspension of very smaller grains was poured into another vessel. A polished silver plate was placed on the bottom of the vessel. After the alcohol evaporated the YBCO powder formed a film on the Ag surface. The film was pressed with the pressure of about $0.3 \mathrm{GPa}$ and then the sintering procedure was repeated for thin film together with the $\mathrm{YBCO}$ pallets at $915^{\circ} \mathrm{C}$ and $925^{\circ} \mathrm{C}$.

\section{Results}

The resistivity and AC susceptibility measurements of YBCO pallets sintered at the temperatures of $925^{\circ} \mathrm{C}$ and $915{ }^{\circ} \mathrm{C}$ are presented on the Fig. 1 and Fig. 2, respectively. They show a good quality of the superconducting material.

The temperature dependencies of the resistance shown in the Fig. 3 exhibit distinct drop at temperatures near $90 \mathrm{~K}$ clearly visible on the significant silver metallic background. The dispersion $\left(\chi^{\prime}\right)$ and the absorption $\left(\chi^{\prime \prime}\right)$ 


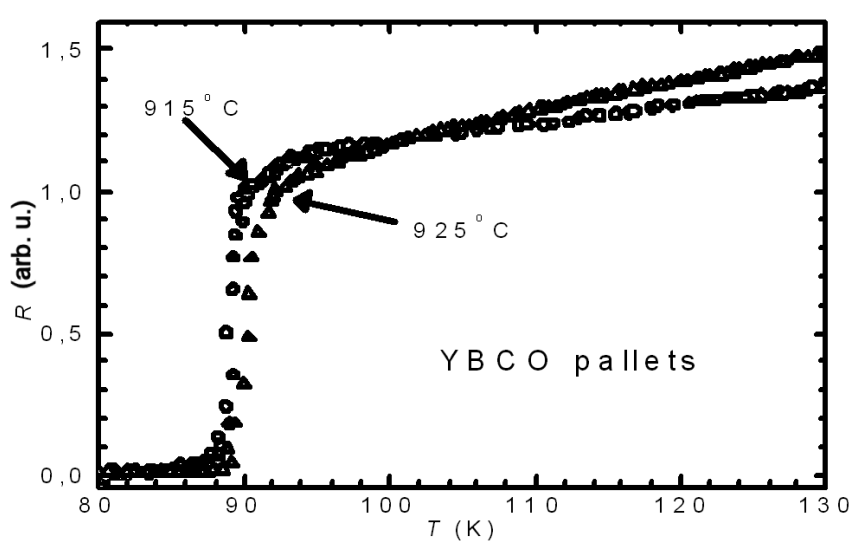

Fig. 1. Resistance vs. temperature for $\mathrm{YBa}_{2} \mathrm{Cu}_{3} \mathrm{O}_{\delta}$ pallets sintered at temperatures as indicated.

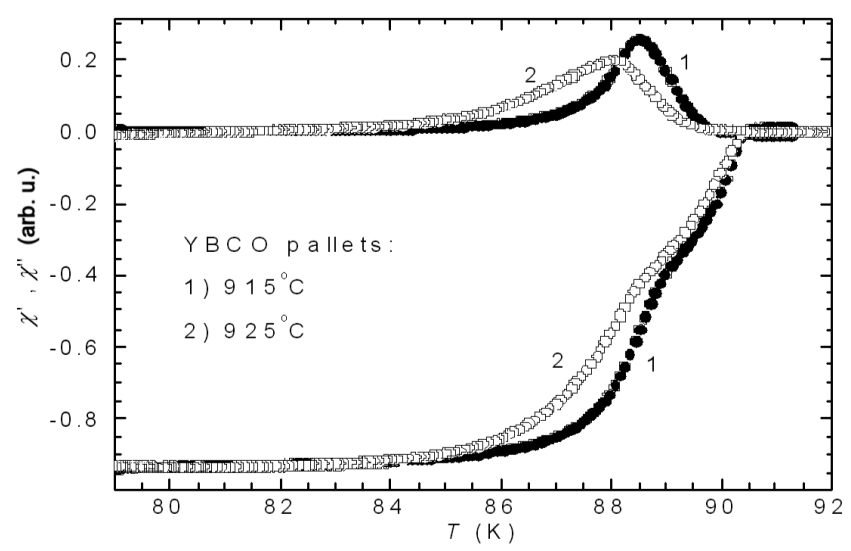

Fig. 2. Temperature dependences of AC susceptibility taken at 0.5 Oe $H_{a c}$ amplitude of the $\mathrm{YBa}_{2} \mathrm{Cu}_{3} \mathrm{O}_{\delta}$ pallets sintered at temperatures as indicated.

parts of AC susceptibility measurements presented in Figs. 4. and 5 assure that the films do not form separate superconducting islands but they are rather superconducting in the whole volume. The absorption part of the AC susceptibility exhibits in general two peaks: the small one close to the critical temperature which is connected with the absorption processes in the grains (see

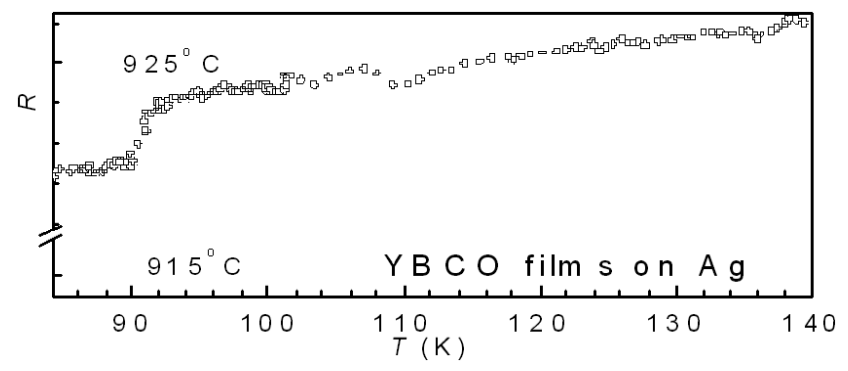

Fig. 3. Resistance vs. temperature for the YBCO films deposited on the silver substrate. the arrow in Fig. 4) that does not practically depend on the applied magnetic field. The second, dominating peak concerns to the inter-granular regions moves fast to the lower temperatures when the applied magnetic field is increased. The temperature dependencies of the dispersion parts of AC susceptibility do not depend on AC magnetic field amplitude in the vicinity of the critical temperature and these curves are split at the temperature below about $90.3 \mathrm{~K}$

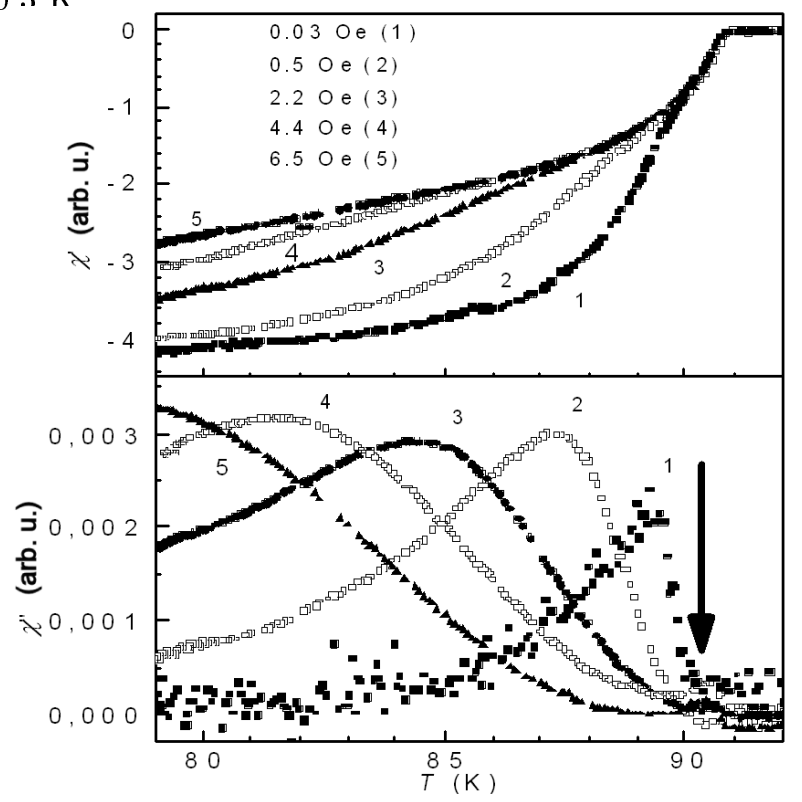

Fig. 4. Temperature dependences of AC susceptibility for YBCO film sintered at $915^{\circ} \mathrm{C}$, measured at different $H_{a c}$ amplitudes. The arrow shows the intra-granular peaks that practically do not depend on the applied magnetic field.

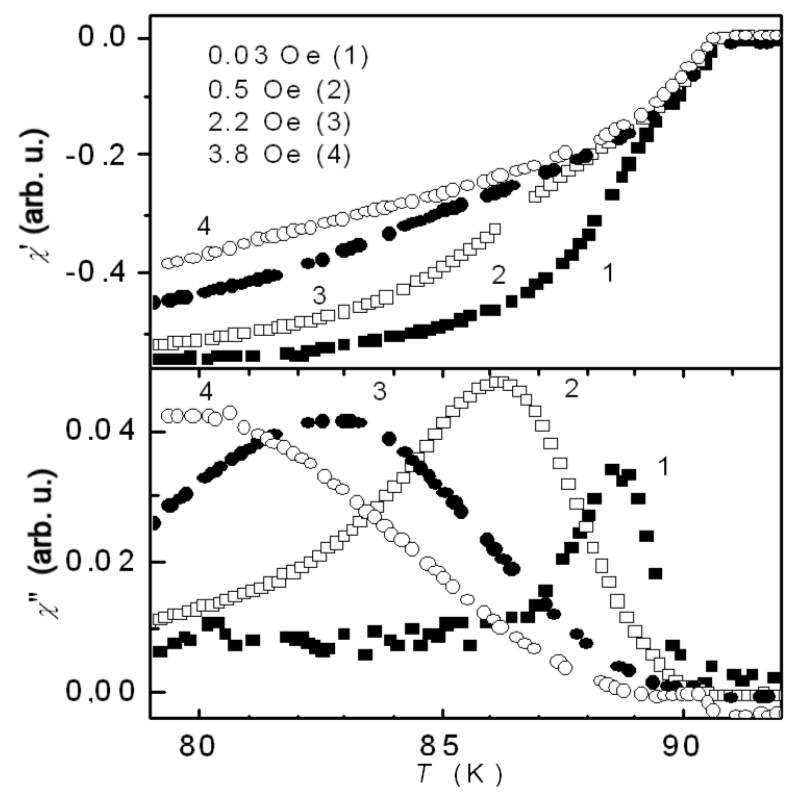

Fig. 5. Temperature dependences of AC susceptibility for YBCO film sintered at $925^{\circ} \mathrm{C}$, measured at different $H_{a c}$ amplitudes. 
These measurements show that we do not loose the superconducting properties in consequence of deposition superconducting powder onto Ag surface and sintering it together. From the susceptibility measurements the intergrain critical temperature defined at the maximum $\chi^{\prime \prime}\left(H_{a c} \rightarrow 0\right)$ is about $88-89 \mathrm{~K}$ while the intra-grain critical temperature defined for $\chi^{\prime}=0$ is only about $1 \mathrm{~K}$ higher.

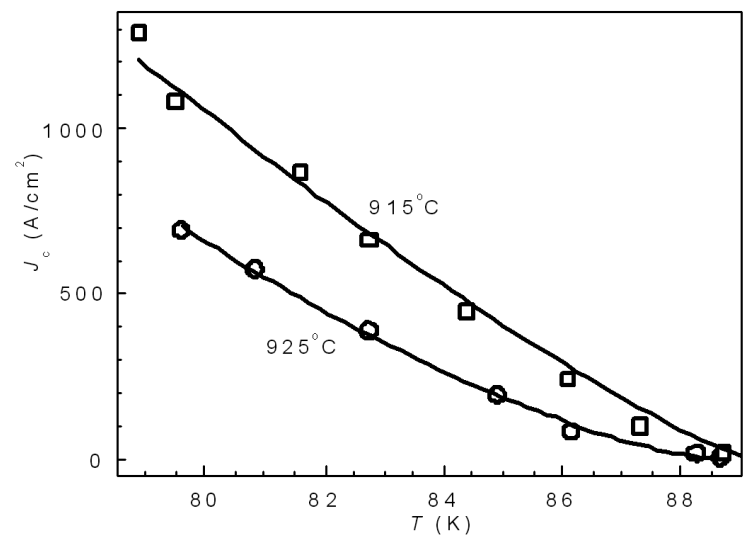

Fig. 6. Intergrain critical current densities versus temperature of the $\mathrm{YBa}_{2} \mathrm{Cu}_{3} \mathrm{O}_{\delta}$ films deposited on $\mathrm{Ag}$, sintered at $915{ }^{\circ} \mathrm{C}$ and $925^{\circ} \mathrm{C}$. Solid lines are the fit to Eq. (2).

The temperature dependence measurements of the absorption part of AC susceptibility at different $H_{a c}$ amplitudes were carried out to evaluate the critical current

densities of the films. They were obtained from the peak position of the absorption part of susceptibility within the Bean's critical state model from the formula $[7,8]$ :

$$
J_{\mathrm{c}}=\frac{2 H_{a c}}{d},
$$

where $H_{a c}$ is the $\mathrm{AC}$ magnetic field amplitude and $d$ is the sample thickness in the perpendicular direction to the AC field. The results of inter-grain critical current densities calculations for the films sintered at $915^{\circ} \mathrm{C}$ and at $925^{\circ} \mathrm{C}$ are presented on the Fig. 6 .

The critical current flows through the inter-grain links which may be treated as Josephson junctions (weak links). The critical current is limited by the weakest junctions on the percolation path of the current. According to the Ginzburg-Landau strong coupling limit approach, the critical current varies with temperature in the following manner [9]:

$$
J_{\mathrm{c}}=J_{\mathrm{c} 0}\left(1-\frac{T}{T_{\mathrm{c}}}\right)^{n},
$$

where $T_{\mathrm{c}}$ is the critical temperature that depends on the applied magnetic field and $J_{\mathrm{c} 0}$ is the critical current at $0 \mathrm{~K}$. Originally, the exponent $n$ was determined to be 1.5 [10] but it may vary within a wide range [11]. If the exponent $n$ is greater than unity there is a strong pinning and the vortex glass structure exists. In this case the function expressed by Eq. (2) has an upward curvature which is typical for HTS. If the exponent $n$ is less than unity the pinning force is weak, which limits the critical current. The function expressed by Eq. (2) has then a downward curvature typical for low temperature superconductors.

TABLE

critical current densities for the YBCO films and bulk samples. The fitting parameters are defined in the Eq. (2).

\begin{tabular}{l|c|c|c|c}
\hline \hline \multicolumn{1}{c|}{ sample } & thickness $[\mathrm{mm}]$ & $J_{\mathrm{c} 0}\left[\mathrm{kA} / \mathrm{cm}^{2}\right]$ & $T_{\mathrm{c}}[\mathrm{K}]$ & $n$ \\
\hline $\begin{array}{l}\text { YBCO film on } \mathrm{Ag} \\
\text { sintered at } 915 \mathrm{C}\end{array}$ & $0.08 \pm 0.01$ & $16 \pm 5$ & $89.2 \pm 0.6$ & $1.21 \pm 0.16$ \\
$\begin{array}{l}\text { YBCO film on } \mathrm{Ag} \\
\text { sintered at } 925^{\circ} \mathrm{C}\end{array}$ & $0.09 \pm 0.01$ & $20 \pm 5$ & $88.6 \pm 0.4$ & $1.46 \pm 0.14$ \\
\hline $\begin{array}{l}\text { YBCO pallet sin- } \\
\text { tered at } 915^{\circ} \mathrm{C}\end{array}$ & $1.50 \pm 0.05$ & $2.6 \pm 0.9$ & $89.1 \pm 0.3$ & $1.16 \pm 0.15$ \\
$\begin{array}{l}\text { YBCO pallet sin- } \\
\text { tered at } 925^{\circ} \mathrm{C}\end{array}$ & $1.15 \pm 0.05$ & $2.7 \pm 0.5$ & $88.6 \pm 0.2$ & $1.15 \pm 0.08$
\end{tabular}

The temperature dependencies of the critical current densities of the films were fitted using Eq. (2) and these fits are shown in Fig. 6 (solid lines). The fit parameters: $J_{\mathrm{c} 0}, T_{\mathrm{c}}$ and $n$ for the both YBCO films as well as for the YBCO pallets are collected in the Table. The intergrain

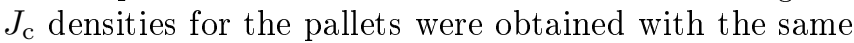
procedure as was described for the films i.e. from the AC susceptibility measurements. The appropriate thickness of the samples are also presented.

From our study we observe the existence of supercon- ductivity up to $90 \mathrm{~K}$ for both YBCO films, but the critical current densities are not very high. The $915^{\circ} \mathrm{C}-\mathrm{YBCO}$ film exhibits slightly higher critical current densities than the film sintered at $925^{\circ} \mathrm{C}$. The fitting parameters for the bulk and film specimens are almost the same.

\section{Summary}

The silver substrate does not influence strongly the properties of YBCO thick films if they are sintered at temperatures of $925^{\circ} \mathrm{C}$ or at lower temperatures. Their 
superconducting parameters are similar to those of the bulk samples and probably could be better if the optimal procedure (such as heat treatment, appropriate pressures and other) would be elaborated.

\section{Acknowledgments}

This work was supported by the Polish Ministry of Science and Higher Education and its grants for Scientific Research. One of us (M. Ch.) has been partly supported by the EU Human Capital Operation Program, Polish Project No. POKL.04.0101-00-434/08-00.

\section{References}

[1] B.A. Głowacki, J. Jackiewicz, J. Appl. Phys. 75, 2993 (1994).

[2] R. Inada, Y. Iwata, K. Tateyama, Y. Nakamura, A. Oota, P.X. Zhang, Physica C 445-448, 762 (2006).

[3] S. Ochiai, D. Doko, H. Rokkaku, M. Fujimoto, H. Okuda, M. Hojo, M. Tanaka, M. Sugano, K. Osamura, M. Mimura, Physica C 445-448, 746 (2006).
[4] Y. Ma, K. Watanabe, S. Awaji, M. Motokawa, Physica C 357-360, 337 (2001).

[5] W.M. Woch, J. Chmist, J. Przewoźnik, J. Niewolski, R. Zalecki, A. Kołodziejczyk, Mol. Phys. Rep. 34/1, 175 (2001).

[6] T. Kato, S. Kobayashi, K. Yamazaki, K. Ohkura, M. Ueyama, N. Ayai, J. Fujikami, E. Ueno, M. Kikuchi, K. Hayashi, K. Sato, Physica C 412414, 1066 (2004).

[7] c.P. Bean, Phys. Rev. Lett. 8, 250 (1962).

[8] J.R. Clem, Physica C 153-155, 50 (1988).

[9] J.R. Clem, B. Bumble, S.I. Raider, W.J. Gallagher, Y.C. Shih, Phys. Rev. B 35, 7526 (1987).

[10] K.A. Müller, M. Takashige, J. Bednorz, Phys. Rev. Lett. 58, 1143 (1987); Y. Yeshurun, A.P. Malozemoff, Phys. Rev. Lett. 60, 2202 (1988).

[11] W.M. Woch, W. Tokarz, R. Zalecki, A. Kołodziejczyk, C. Deinhofer, G. Gritzner, Supercond. Sci. Technol. 23, 025004 (2010) 